\title{
There is no uniformly unbiased independence test for densities
}

\author{
Jonas Moss \\ Department of Mathematics, University of Oslo, PB 1053, Blindern, NO-0316, Oslo, Norway
}

\begin{abstract}
There is no uniformly unbiased independence test for densities.

\section{Introduction}

Is there an uniformly unbiased independence test for densities? This question can be traced back to Hoeffding (1948), who proved the non-existence of uniformly unbiased independence tests based on ranks. As a test that fails to be uniformly unbiased has no power against at least one alternative, uniform unbiasedness is a property any sensible test should satisfy.

In the tradition of Bahadur and Savage (1956) and Romano (2004), Shah and Peters (2018) recently showed a strong impossibility result for conditional independence testing. In this case there is no test that is unbiased against any alternative, a property Shah and Peters (2018) and termed untestable. This is not true for unconditional independence testing as there are tests such as Hoeffding's rank test (Hoeffding, 1948) with pointwise asymptotic power equal to 1.
\end{abstract}

\section{The Result}

Let $\mathcal{P}$ and $\mathcal{Q}$ be two families of probabilities. Consider the sequence of testing problems

$$
\begin{array}{ll}
H_{0}: & P^{n} \in \mathcal{P}^{n} \\
H_{a} & : \quad Q^{n} \in \mathcal{Q}^{n}
\end{array}
$$

Here $P^{n}$ is the product of $n$ probability measures $P$. This corresponds to independent and identical sampling of $n$ points from $P$. A test with level $\alpha$ is a sequence of sets $\left(A_{1}, A_{2}, \ldots\right)$

Email address: jonasmgj@math.uio.no (Jonas Moss) 
satisfying $P^{n}\left(A_{n}\right) \geq 1-\alpha$ for all $n$. The sets $A_{n}$ are the acceptance sets for $H_{0}$. A test is biased if there for every $n$ is a member $Q \in \mathcal{Q}$ satisfying $Q^{n}\left(A_{n}\right) \geq 1-\alpha$. A test is uniformly unbiased if it is not biased. These definitions are straightforward adaptations of the classical definitions found in e.g. Lehmann and Romano (2008).

All mentions of "almost everywhere" are with respect to the Lebesgue measure.

Theorem 1. There is no uniformly unbiased test of independence of any level for densities on $\mathbb{R}^{k}$, $k \geq 2$.

Let $\mathcal{P}$ be the family of independent densities, $\mathcal{Q}$ be the family of dependent densities, and $A_{n}$ a set satisfying $P^{n}\left(A_{n}\right) \geq 1-\alpha$ for all $P \in \mathcal{P}$. Let $I \in \Sigma$ be a hypercube in $\mathbb{R}^{k}$. Then there is a $Q \in \mathcal{Q}$ that fails to be independent on $I$, for instance when $Q$ equals the diagonal of $I$. Choose an $n \in \mathbb{N}$ and take a look at $I^{n} \cap A_{n}$, where one of three things can happen.

(i) The intersection is empty almost everywhere. Now choose a $Q \in Q$ which equals a $P \in \mathcal{P}$ everywhere except $I$. Then $Q^{n}\left(A_{n}\right) \geq 1-\alpha$, and the test is biased.

(ii) The intersection equals $I^{n}$ almost everywhere. Again choose a $Q \in Q$ which equals some $P \in \mathcal{P}$ everywhere except $I$. Then $Q^{n}\left(A_{n}\right) \geq 1-\alpha$, and the test is biased.

(iii) The intersection is something else entirely. That is, both $I^{n} \cap A_{n} \neq \emptyset$ and $I^{n} \cap A_{n} \neq I^{n}$ are true almost everywhere.

If (i) or (ii) holds the test $A_{n}$ is biased, so we will have to take a look at case (iii). For the test to have a chance of being unbiased, this condition has to hold for all hypercubes $I$.

The family $\mathcal{P}$ includes the uniform distribution on $I$ for every hypercube $I$. A uniform distribution has probability measure $P(B)=\lambda(B \cap I) / \lambda(I)$. Since $P\left(A_{n}\right) \geq 1-\alpha$ for all $P \in \mathcal{P}$, $\lambda\left(A_{n} \cap I\right) / \lambda(I) \geq 1-\alpha$. But then $\lambda\left(A^{c}\right)=0$ by the following lemma.

Lemma 2. Let $A$ be Lebesgue measurable. If there is a real number $c>0$ such that $\lambda(A \cap U) \geq$ $c \lambda(U)$ for every hypercube $U$ then $A^{c}$ has measure 0.

Proof. By assumption

$$
\lambda\left(A^{c} \cap U\right) \leq(1-c) \lambda(U)
$$

for each $U$. By Lebesgue's density theorem (Rudin, 1986, Theorem 7.10), when $U_{x}$ is a hypercube centered on $x, \lambda\left(A^{c} \cap U_{x}\right) / \lambda\left(U_{x}\right)$ converges to 1 as $\lambda\left(U_{x}\right) \rightarrow 0$ for almost all $x \in A^{c}$. But 
$\lambda\left(A^{c} \cap U_{x}\right) / \lambda\left(U_{x}\right) \leq 1-c$, so $\lambda\left(A^{c} \cap U_{x}\right) / \lambda\left(U_{x}\right)$ fails to converge to 1 for all $x \in A^{c}$. Hence $A^{c}$ has measure 0 .

If $\lambda\left(A^{c}\right)=0$ then condition (ii) holds for every $I$. This implies the test is biased and Theorem 1 follows.

\section{Acknowledgement}

Thanks to Olav Dovland for helpful comments.

\section{References}

Bahadur, R.R., Savage, L.J., 1956. The nonexistence of certain statistical procedures in nonparametric problems. The Annals of Mathematical Statistics 27, 1115-1122.

Hoeffding, W., 1948. A non-parametric test of independence. The Annals of Mathematical Statistics $19,546-557$.

Lehmann, E.L., Romano, J.P., 2008. Testing Statistical Hypotheses. Springer Science + Business Media.

Romano, J.P., 2004. On non-parametric testing, the uniform behaviour of the t-test, and related problems. Scandinavian Journal of Statistics 31, 567-584.

Rudin, W., 1986. Real and Complex Analysis. McGraw-Hill.

Shah, R.D., Peters, J., 2018. The hardness of conditional independence testing and the generalised covariance measure. arXiv preprint arXiv:1804.07203 . 